TRANSACTIONS OF THE

AMERICAN MATHEMATICAL SOCIETY

Volume 350, Number 4, April 1998, Pages 1703-1712

S 0002-9947(98)02064-9

\title{
A NEW DEGREE BOUND FOR VECTOR INVARIANTS OF SYMMETRIC GROUPS
}

\author{
P. FLEISCHMANN
}

\begin{abstract}
Let $R$ be a commutative ring, $V$ a finitely generated free $R$-module and $G \leq G L_{R}(V)$ a finite group acting naturally on the graded symmetric algebra $A=S(V)$. Let $\beta(V, G)$ denote the minimal number $m$, such that the ring $A^{G}$ of invariants can be generated by finitely many elements of degree at most $m$.

For $G=\Sigma_{n}$ and $V(n, k)$, the $k$-fold direct sum of the natural permutation module, one knows that $\beta\left(V(n, k), \Sigma_{n}\right) \leq n$, provided that $n$ ! is invertible in $R$. This was used by E. Noether to prove $\beta(V, G) \leq|G|$ if $|G| ! \in R^{*}$.

In this paper we prove $\beta\left(V(n, k), \Sigma_{n}\right) \leq \max \{n, k(n-1)\}$ for arbitrary commutative rings $R$ and show equality for $n=p^{s}$ a prime power and $R=\mathbb{Z}$ or any ring with $n \cdot 1_{R}=0$. Our results imply

$$
\beta(V, G) \leq \max \{|G|, \operatorname{rank}(V)(|G|-1)\}
$$

for any ring with $|G| \in R^{*}$.
\end{abstract}

\section{INTRODUCTION}

Let $R$ be a commutative ring, $V$ a finitely generated free $R$-module and $G \leq$ $G L_{R}(V)$ a finite group. Then $G$ acts naturally on the symmetric graded algebra

$$
A:=S_{R}(V)=\bigoplus_{i=0}^{\infty} S_{R}(V)_{i},
$$

where $S_{R}(V)_{i}$ denotes the $i$-th symmetric power of $V$. Notice that the action of $G$ preserves degrees; hence the algebra $A^{G}$ of invariants inherits the grading from $A$.

It has been a classical problem of $19^{\text {th }}$ century algebra to construct minimal sets of generators for the ring of invariants $A^{G}$ or sets of generators with minimal degree.

In [3] (1916) E. Noether gave two different constructive proofs for the fact that $A^{G}$ is finitely generated by invariants of degree at most $|G|$, if $\mathbb{Q} \subseteq R$. Both proofs can be made to work over any ring $R$ having the property that the factorial $|G|$ ! of the group order is invertible (e.g. see [7], [2]).

In [4] (1926), Noether presented a proof for the fact that $A^{G}$ is finitely generated whenever $R$ is a noetherian ring. No assumption whatsoever on invertibility of $|G|$ is needed, but the price one has to pay for this generality is that the proof is no longer constructive. It essentially uses the fact that submodules of noetherian modules are finitely generated. The first proof in [3] uses a classical result of $\mathrm{H}$.

Received by the editors June 20, 1996.

1991 Mathematics Subject Classification. Primary 13A50.

(C)1998 American Mathematical Society 
Weyl [8] on vector invariants of the symmetric groups. To state this and to show how it is applied, we have to introduce some notation:

Let $V:=V(n)=R^{n}$ be the free $R$-module with the natural permutation action of $\Sigma_{n}$ on it. For $k \in \mathbb{N}$ we define $V(n, k):=V^{\oplus k}=V \oplus \cdots \oplus V$ to be the $k$-fold direct sum with diagonal action of $\Sigma_{n}$ and $A_{R}(n, k):=S(V(n, k))$ to be the symmetric $R$-algebra over $V(n, k)$. Let $\left\{x_{1}, \ldots, x_{n}\right\}$ be a basis of $V(n)$; then for $k \in \mathbb{N}$ the set $\left\{x_{11}, \ldots, x_{n 1}, \ldots, x_{1 k}, \ldots, x_{n k}\right\}$ is a basis of $V^{\oplus k}$.

A monomial $X:=x_{11}^{a_{11}} \cdot \ldots \cdot x_{n 1}^{a_{n 1}} \cdot \ldots \cdot x_{1 k}^{a_{1 k}} \cdot \ldots \cdot x_{n k}^{a_{n k}} \in S\left(V^{\oplus k}\right)$ is of multidegree $\operatorname{md}(X):=\left(d_{1}, \ldots, d_{k}\right) \in \mathbb{N}^{k}$ if $d_{j}=\sum_{i=1}^{n} a_{i j}$ and of (total) degree $d(X):=\sum_{j=1}^{k} d_{j}$. Clearly $A_{R}(n, k)=\bigoplus_{\underline{m} \in \mathbb{N}^{k}} A_{R}(n, k)_{\underline{m}}$, where $A_{R}(n, k)_{\underline{m}}$ is the set of $R$-linear combinations of monomials with multidegree $\underline{m}$ and the $A_{R}(n, k)_{m}$ 's are $\Sigma_{n}$-submodules. An element of $A_{R}(n, k)_{\underline{a}}$ is called homogeneous of multidegree $\underline{m}$.

Now let $G=\left\{e=g_{1}, g_{2}, \ldots, g_{n}\right\}$ be an arbitrary finite group of order $n$ and let $W=R w_{1} \oplus \cdots \oplus R w_{k}$ be an $R G$-module of rank $k$. Consider the Cayley embedding $G \hookrightarrow \Sigma_{n}, g \mapsto\left(g_{i} \mapsto g_{j}:=g g_{i}\right)$. Then

$$
\nu: A_{R}(n, k) \rightarrow S_{R}(W), \quad x_{i \ell} \mapsto g_{i}\left(w_{\ell}\right)
$$

is a $G$-equivariant algebra epimorphism which preserves degrees. In fact,

$$
\nu\left(g\left(x_{i \ell}\right)\right)=\nu\left(x_{j \ell}\right)=g_{j}\left(w_{\ell}\right)=g g_{i}\left(w_{\ell}\right)=g \nu\left(x_{i \ell}\right) .
$$

Now, for completeness, we repeat Noether's original 1916 argument showing that the restriction

$$
\nu: A_{R}(n, k)^{\Sigma_{n}} \rightarrow S_{R}(W)^{G}
$$

is surjective whenever $n$ is invertible in $R$ : In this case, for any $f=f\left(w_{1}, \ldots, w_{k}\right) \in$ $S_{R}(V)^{G}$ we can define

$$
F:=\frac{1}{n}\left(f\left(x_{11}, x_{12}, \ldots, x_{1 k}\right)+\cdots+f\left(x_{n 1}, x_{n 2}, \ldots, x_{n k}\right)\right) \in A_{R}(n, k)^{\Sigma_{n}}
$$

and get

$$
\begin{aligned}
\nu(F) & =\frac{1}{n}\left(f\left(g_{1}\left(w_{1}\right), g_{1}\left(w_{2}\right), \ldots, g_{1}\left(w_{k}\right)\right)+\cdots+f\left(g_{n}\left(w_{1}\right), g_{n}\left(w_{2}\right), \ldots, g_{n}\left(w_{k}\right)\right)\right. \\
& =\frac{1}{n}\left(g_{1} f\left(w_{1}, w_{2}, \ldots, w_{k}\right)+\cdots+g_{n} f\left(w_{1}, w_{2}, \ldots, w_{k}\right)=f\right.
\end{aligned}
$$

Let $\beta(W, G)$ denote the minimum number $m$ such that $S_{R}(W)^{G}$ is generated by finitely many invariants of degree at most $m$ and $\beta_{R}(G):=\max \{\beta(W, G)\}$, where $W$ ranges through all $R G$-modules that are finitely generated and free as $R$-modules. We see immediately that $|G| \in R^{*}$ implies

$$
\beta(W, G) \leq \beta\left(V(n, k), \Sigma_{n}\right) .
$$

For any $f=f\left(x_{1}, \ldots, x_{n}\right) \in S(V(n))$ and $\underline{m}=\left(m_{1}, \ldots, m_{k}\right) \in \mathbb{N}^{k}$ we define $\operatorname{Pol}(f)_{\underline{m}}$ to be the $A_{R}(n, k)_{\underline{m}}$-part of the element

$$
f\left(x_{11}+\cdots+x_{1 k}, \ldots, x_{n 1}+\cdots+x_{n k}\right) .
$$

Notice that $\operatorname{Pol}(f)_{\underline{m}} \in A_{R}(n, k)^{G}$ if $f \in S(V(n))^{G}$ for any $G \leq \Sigma_{n}$; moreover $\operatorname{Pol}(f)_{\underline{m}}=0$ whenever $\sum_{i} m_{i}$ exceedes the degree of $f \in S(V(n))$.

Now Weyl's theorem states that, if $R=K$ is a field containing $\mathbb{Q}$, then

$$
A_{K}(n, k)^{\Sigma_{n}}=K\left[\operatorname{Pol}\left(e_{i}\right)_{\underline{m}} \mid i=1, \ldots, n, \underline{m} \in \mathbb{N}^{k}\right],
$$


where $e_{i}$ is the $i$-th elementary-symmetric polynomial. Since $\left(e_{i}\right)_{\underline{m}}=0$ if $\underline{m}=$ $\left(m_{1}, \ldots, m_{k}\right)$ and $\sum_{j} m_{j}>i, A_{K}(n, k)^{\Sigma_{n}}$ is generated by a finite number of elements of degree $\leq n$.

Recently, new proofs for this result have been given by D. Richman and others, which work over any commutative ring $R$ with $n ! \in R^{*}$ (see [2]), In this case Weyl's theorem implies $\beta\left(V(n, k), \Sigma_{n}\right) \leq n=|G|$; hence $\beta_{R}(G) \leq|G|$.

But, as we will see, Weyl's theorem no longer holds in positive characteristic $\leq n$. Hence although the restriction $\nu: A_{R}(n, k)^{\Sigma_{n}} \rightarrow S_{R}(V)^{G}$ is surjective in the more general situation where only $|G|$ is invertible in $R$, a proof of $\beta_{R}(G) \leq|G|$ in this generality is not known at the moment of this writing (see [2] and [6] for proofs in the case of solvable groups).

It is the aim of this paper to analyze vector invariants of $\Sigma_{n}$ in the situation where $R$ is an arbitrary commutative ring for example the ring of rational integers $\mathbb{Z}$.

In [1], Campbell, Hughes and Pollack prove $\beta\left(V(n, k), \Sigma_{n}\right) \leq \max \left\{|G|, k \cdot\left(\begin{array}{l}n \\ 2\end{array}\right)\right\}$, and thus obtain $\beta(V, G) \leq \max \left\{|G|, \operatorname{rank}(V) \cdot\left(\begin{array}{c}|G| \\ 2\end{array}\right)\right\}$ for any commutative ring $R$ with $|G| \in R^{*}$.

I will prove that $\beta\left(V(n, k), \Sigma_{n}\right) \leq \max \{n, k(n-1)\}$ for arbitrary commutative rings $R$, with equality if $n=p^{s}$, a prime power, and $R=\mathbb{Z}$ or $p \cdot 1_{R}=0$. This will imply

$$
\beta(V, G) \leq \max \{|G|, \operatorname{rank}(V)(|G|-1)\}
$$

if $|G|$ is invertible in $R$. It also implies, for the 'global bounds' over $R=\mathbb{Z}$ or $R=K$, a field of characteristic $p$,

$$
\beta_{R}\left(\Sigma_{p^{s}}\right)=\infty
$$

Hence in this case there is no finite global bound at all, even for the smallest nontrivial group $\Sigma_{2}$.

\section{Multiplication IN INVARIANT RINGS}

Let $R, V, A$ and $G$ be as in the introduction and $U \leq G$ a subgroup. Then we have the following homomorphism of $R$-modules:

$$
T_{U}^{G}: A^{U} \rightarrow A^{G}, a \mapsto \sum_{g \in[G: U]}{ }^{g} a,
$$

where $[G: U]$ denotes an arbitrary cross-section of (left) $U$-cosets in $G$, i.e. $G=$ $\biguplus_{g \in[G: U]} g U$. This is called the (relative) trace homomorphism. It preserves (multi)degrees and if the index $|[G: U]|$ is invertible in $R$, then $T_{U}^{G}$ is an epimorphism. (Since $a \in A^{U}$, its relative trace $T_{U}^{G}(a)$ does not depend on the choice of coset representatives).

We will give a 'Mackey formula' for the product of relative traces which is particularly useful in the case of invariant rings of permutation modules.

Let $U, V \leq G$ be subgroups and suppose we are given a a cross-section $[U: G: V]$ of $U, V$-double cosets with $G=\biguplus_{g \in[U: G: V]} U g V$ and for each $g \in[U: G: V]$ a crosssection $[U g V: V] \subseteq U$ of $V$-cosets with $U g V=\biguplus_{t \in[U g V: V]} t g V$. Then $[U g V: V]$ is also a cross-section $\left[U: U \cap{ }^{g} V\right]$ of $U \cap{ }^{g} V$-cosets in $U$, i.e. $U=\biguplus_{t \in[U g V: V]} t U \cap{ }^{g} V$. Here, as in the following, ${ }^{g} V$ denotes $g V g^{-1}$. 
Proposition 2.1. Let $U, V \leq G$ be subgroups and $[U: G: V]$ be an arbitrary cross-section of $U, V$-double cosets in $G$. Then for each $a \in A^{U}$ and $b \in A^{V}$ we have

$$
T_{U}^{G}(a) \cdot T_{V}^{G}(b)=\sum_{g \in[U: G: V]} T_{U \cap{ }^{g} V}^{G}(a g b) .
$$

Proof. First notice that the summands on the right hand side do not depend on the choice of double coset representatives. Indeed if $u \in U$ and $v \in V$, then we have

$$
T_{U \cap{ }^{u g v} V}^{G}(a(u g v b))=T_{U \cap{ }^{u g} V}^{G}((u a)(u g b))=T_{U \cap{ }^{u g} V}^{G}(u(a g b))=T_{U \cap{ }^{g} V}^{G}(a g b) .
$$

Now we choose $[U: G: V]$ and $[U g V: V]$ such that $G=\biguplus_{g \in[U: G: V], t \in\left[U: U \cap{ }^{g} V\right]} t g V$ and conclude that

$$
\begin{aligned}
T_{U}^{G}(a) \cdot T_{V}^{G}(b) & =T_{U}^{G}\left(a \cdot T_{V}^{G}(b)\right)=\sum_{d \in[G: U], h \in[G: V]} d[a(h b)] \\
& =\sum_{g \in[U: G: V], t \in\left[U: U \cap{ }^{g} V\right], d \in[G: U]} d[a(t g b)] \\
& =\sum_{g \in[U: G: V], t \in\left[U: U \cap{ }^{g} V\right], d \in[G: U]} d t[a(g b)] \\
& =\sum_{g \in[U: G: V]} T_{U \cap{ }^{g} V}^{G}(a(g b)) .
\end{aligned}
$$

From now on we suppose that $G \leq \Sigma_{n}$ and $V:=V(n)$, i.e. that $V$ is a permutation module of $G$. It is well known that in this case the orbit sums of monomials form a free $R$-basis of $A^{G}$, so the formulae above, applied to momomials, completely describe multiplication in $A^{G}$.

More precisely, for any monomial $a \in A$ let $G_{a}$ denote the stablizer $G_{a}:=\{g \in$ $\left.\left.G\right|^{g} a=a\right\}$ and $a_{G}^{+}$denote the $G$-orbit sum $\sum_{g \in\left[G: G_{a}\right]}{ }^{g} a$. (We write $a^{+}$if $G$ is clear from the context.) Then we have

\section{Corollary 2.2.}

$$
a_{G}^{+} \cdot b_{G}^{+}=\sum_{g \in\left[G_{a}: G: G_{b}\right]} \frac{\left|G_{a g b}\right|}{\left|G_{a} \cap{ }^{g} G_{b}\right|}(a g(b))_{G}^{+} .
$$

\section{THE 'ROW-LEX' ORDER OF MATRICES}

Let the set of $k$-vectors over $\mathbb{N}$ be ordered lexicographically, i.e. by the rule $\underline{a}=\left(a_{1}, a_{2}, \ldots, a_{k}\right)<\underline{b}=\left(b_{1}, b_{2}, \ldots, b_{k}\right)$ if and only if there is $1 \leq j_{0} \leq k$ with $a_{j}=b_{j}$ for $j<j_{0}$ and $a_{j_{0}}<b_{j_{0}}$. We say that $\underline{b}$ (strictly) dominates $\underline{a}$ iff $a_{i} \leq b_{i}$ for all $i=1, \ldots, k$, with at least one $a_{i}<b_{i}$. Notice that $\underline{a}<\underline{b}$ whenever $\underline{b}$ strictly dominates $\underline{a}$, but $\underline{a}<\underline{b}$ does not imply that $\underline{a}$ is dominated by $\underline{b}$ (e.g. $(1,2,2)<(2,1,1))$. We will identify the set $\mathcal{M}:=\mathcal{M}(n, k)$ of monomials in $A:=A_{R}(n, k)$ with the set $\operatorname{Mat}_{n, k}\left(\mathbb{N}_{0}\right)$ of nonnegative $n, k$-matrices using the bijection

$$
c: \mathcal{M}(n, k) \rightarrow \operatorname{Mat}_{n, k}\left(\mathbb{N}_{0}\right), x_{11}^{a_{11}} \cdot \ldots \cdot x_{n 1}^{a_{n 1}} \cdot \ldots \cdot x_{1 k}^{a_{1 k}} \cdot \ldots \cdot x_{n k}^{a_{n k}} \mapsto\left(a_{i j}\right) .
$$

Now we define a total order on the set $\mathcal{M}:=\mathcal{M}(n, k)$ of monomials in the following way (we call this the 'row-lex' order): $a=\left(a_{i j}\right)<b=\left(b_{i j}\right)$ if and only if $d(a)<d(b)$ 
or $d(a)=d(b)$ and $m d(a)<m d(b)$ or $m d(a)=m d(b)$ and there is a row index $i_{0} \in \mathbb{N}$ such that the row vectors $a_{i}=b_{i}$ for all $i<i_{0}$ and $a_{i_{0}}<b_{i_{0}}$. Hence the matrices are ordered first by total degree, then multidegree and, if these coincide by the 'row-lexicographic' order. This amounts to the total order of variables $x_{i j}$ given by $x_{11}>x_{21}>\cdots>x_{n 1}>x_{12}>x_{22} \cdots>x_{(n-1) n}>x_{n n}$. Notice that the action of $\Sigma_{n}$ on $\mathcal{M}$ corresponds to the permutation of rows of $a=\left(a_{i j}\right)$, i.e. ${ }^{\sigma} a:=$ $\left(a_{\sigma^{-1}(i), j}\right)=\hat{\sigma} \circ\left(a_{i j}\right)$, where $\hat{\sigma}:={ }^{\sigma}\left(\delta_{i j}\right)$ is the permutation matrix corresponding to $\sigma \in \Sigma_{n}$. Moreover the product of monomials is given by componentwise addition of matrix entries:

$$
a b=c=\left(c_{i j}\right) \text { with } c_{i j}=a_{i j}+b_{i j} ;
$$

hence $m d(a b)=m d(a)+m d(b)$. For $a, b, c \in \mathcal{M}$ we have $a b \geq a$ with equality if and only if $b=1$ (the zero matrix), and $a<b \Longleftrightarrow a c<b c$. For $f=\sum_{a \in \mathcal{M}} r_{a} a \in A$ we define $H T(f)$ to be the highest monomial $a$ with respect to " $<$ " that appears in $f$ with nonzero coefficient $r_{a}$. Now we get a preorder (i.e. a transitive relation) on $A$ by defining $f$ to be less than $g \in A(f \prec g)$ if and only if $H T(f)<H T(g)$. We also define $f \preceq g \Longleftrightarrow f=g$ or $f \prec g$. For $a \in \mathcal{M}$ the orbit sum $a^{+}$will usually be written by displaying its highest element, $a=\max a^{G}$. We get immediately:

Lemma 3.1. Let $a, b \in \mathcal{M}$ with $a:=\max a^{G}$ and $b:=\max b^{G}$. Then $G_{a b}=$ $G_{a} \cap G_{b}$ and

$$
(a b)^{+}-a^{+} b^{+} \prec(a b)^{+} .
$$

Proof. First note that $G_{a} \cap G_{b} \subseteq G_{a b}$, so we only have to prove the opposite inclusion. Suppose then that $g \in G$ but $g \notin G_{a} \cap G_{b}$. Then without loss of generality we may assume $g \notin G_{a}$. Hence $g a \prec a$ and $g(a b)=g(a) g(b) \prec a b$. Therefore $g \notin G_{a b}$, and so $G_{a b}=G_{a} \cap G_{b}$. Obviously the lead term of $a^{+} b^{+}$is $a b$; to finish the proof of the lemma it suffices to show that $a b$ is also the lead term of $(a b)^{+}$. Suppose $g \in G$ with $g \notin G_{a b}$; then we can assume that $g \notin G_{b}$, and hence $g(a b)=g(a) g(b) \preceq a(g(b)) \prec a b$.

Remark 3.2. Notice that, by 2.2 , we have in fact

$$
(a b)^{+}-a^{+} b^{+}=-\left\{\sum_{g \in\left[G_{a}: G: G_{b}\right] \backslash G_{a} \cdot G_{b}} \frac{\left|G_{a g b}\right|}{\left|G_{a} \cap{ }^{g} G_{b}\right|}(a g(b))^{+}\right\} .
$$

For $j=1, \ldots, k$ let $\underline{1}^{(j)} \in \mathcal{M}$ denote the matrix whose entries are all one in the $j^{\text {th }}$ column and zero elsewhere. Let $a \in \mathcal{M}$ be such that for some column index $j$ and all $i=1, \ldots, n$ the entries $a_{i j}$ are positive. Then

$$
a^{+}=\left(\begin{array}{ccccc}
a_{11} & \ldots & a_{1 j}-1 & \ldots & a_{1 k} \\
a_{21} & \ldots & a_{2 j}-1 & \ldots & a_{2 k} \\
\ldots & \ldots & \ldots & \ldots \ldots & \\
a_{n 1} & \ldots & a_{n j}-1 & \ldots & a_{n k}
\end{array}\right)^{+} \cdot\left(\begin{array}{ccccc}
0 & \ldots & 1 & \ldots & 0 \\
0 & \ldots & 1 & \ldots & 0 \\
\ldots & \ldots & \ldots & \ldots & \\
0 & \ldots & 1 & \ldots & 0
\end{array}\right)^{+} .
$$

Hence $A^{G}$ can be generated by the $\underline{1}^{(i)}$ 's and orbit sums $a^{+}$such that each column of $a \in M$ has a zero entry. 
If $G=\Sigma_{n}$ and $\tilde{a} \in \mathcal{M}$, then $\max \tilde{a}^{G}$ has a particularly easy shape: let $\left(a_{1} \geq\right.$ $\left.a_{2} \geq \cdots \geq a_{n}\right)$ be the family of row vectors of $\tilde{a}$ and consider

$$
b:=\left(\begin{array}{c}
b_{1} \\
b_{2} \\
\cdots \\
b_{n}
\end{array}\right) \neq a:=\left(\begin{array}{c}
a_{1} \\
a_{2} \\
\ldots \\
a_{n}
\end{array}\right),
$$

where the unordered families $\left(a_{i}\right)$ and $\left(b_{i}\right)$ coincide. Let $i_{0}:=\min \left\{i \mid a_{i} \neq b_{i}\right\}$; then the families $\left(a_{i}\right)_{1 \leq i<i_{0}}$ and $\left(b_{i}\right)_{1 \leq i<i_{0}}$ coincide and $b_{i_{0}}$ must be one of the $a_{i}$ 's with $i>i_{0}$; hence $b_{i_{0}}<a_{i_{0}}$ and $b<a$. We see that $a$ is the maximal element in the orbit $a^{\Sigma_{n}}=\tilde{a}^{\Sigma_{n}}$.

\section{INDECOMPOSABLE INVARIANTS}

The following definition as well as the lemma make sense for arbitrary invariant rings of finite groups:

Definition 4.1. For $d \in \mathbb{N}$ let $\mathrm{R}(d):=R\left[b \in A^{G} \mid d(b)<d\right]$. An element $a \in A^{G}$ is called indecomposable if and only if $a \notin \mathrm{R}(d(a))$, and decomposable if $a \in$ $\mathrm{R}(d(a))$. (In particular, 0 is decomposable.)

Lemma 4.2. Let $S \subseteq A^{G}$. Then the following are equivalent:

(i) $A^{G}=R[S]$;

(ii) For each $a \in \mathcal{M}$ with $a^{+} \in A^{G} \backslash S$ there is $f \in R[S]$ with $f=0$ or $d(f) \leq d(a)$ such that $a^{+}-f$ is decomposable.

Proof. (i) $\Rightarrow$ (ii). We can take $f=a^{+}$.

(ii) $\Leftarrow\left(\right.$ i). Let $m \in \mathcal{M}$ be of minimal degree $d$ such that $m^{+} \in A^{G} \backslash R[S]$. Then $m^{+} \notin S$, so $c:=m^{+}+f$ is decomposable for some $f \in R[S]$ of degree $\leq d$ or $f=0$. In any case $d(c) \leq d$ and $c \in \mathrm{R}(d)$, so it is a linear combination of products of elements $b^{+}$of degree $<d$. By minimality of $d$, each of these $b^{+}$'s lies in $R[S]$; hence $c$ and $m^{+}$lie in $R[S]$, a contradiction. So $R[S]=A^{G}$.

\section{Remark 4.3 .}

$$
\beta(V(n, k), G)=\max \left\{d \mid \mathrm{R}(d)<A^{G}\right\}=\min \left\{d \mid \mathrm{R}(d+1)=A^{G}\right\} .
$$

From now on let $G:=\Sigma_{n}$ and $A:=A(n, k)$.

Definition 4.4. Let $\mathcal{B}^{1}$ denote the $R$-module spanned by those elements $b^{+} \in A^{G}$ with $b \in \mathcal{M}$ such that the first column vector $\underline{b}^{1}$ of $b$ lies in $\{0,1\} \underline{n}$ and all rows of $b$ starting with 1 in the first column are of the form $(1,0, \ldots, 0)$.

Lemma 4.5. For any $a \in \mathcal{M}$ we have

$$
a^{+} \in\left(\mathcal{B}^{1} \cap A_{m d(a)}^{G}\right)+\mathrm{R}\left(d\left(a^{+}\right)\right) .
$$

This implies that if $\operatorname{md}(a)=\left(a_{1}, a_{2}, \ldots, a_{k}\right)$ with $a_{1}>n$ or $a_{1}=n$ and $\operatorname{md}(a) \neq$ $(n, 0,0, \ldots, 0)$, then $a^{+}$is decomposable, and if $\operatorname{md}(a)=(n, 0,0, \ldots, 0)$, then $a^{+}-$ $r \underline{1}^{(1)}$ is decomposable for some $r \in R$.

Proof. We use induction with respect to the $\prec$ relation. Let $a=\max a^{G}$ with $d\left(a^{+}\right)=d$ and assume that all $b^{+}$with $b^{+} \prec a^{+}\left(\right.$and $\left.\operatorname{md}(b)=\operatorname{md}(a)=\left(a_{1}, \ldots, a_{k}\right)\right)$ already lie in $\mathcal{B}^{1} \cap A_{m d(a)}^{G}+\mathrm{R}(d)$. We can assume that at least one zero appears in the first column of $a$. Now there are three cases to consider: the first column has 
three distinct entries, two distinct entries with the non-zero entry bigger than one, and two distinct entries with the non-zero entry equal to one.

So we assume first that $a_{11} \geq a_{21} \geq \ldots a_{s 1}>c=a_{(s+1) 1}=\cdots=a_{t 1}>0=$ $a_{(t+1) 1}=\cdots=a_{n 1}$. Consider

$$
a^{+}=\left(\begin{array}{cc}
a_{11} & \underline{b}_{1} \\
a_{21} & \underline{b}_{2} \\
\cdots & \underline{b}_{s} \\
a_{s 1} & \underline{b}_{s} \\
c & \underline{b}_{s+1} \\
c & \underline{b}_{s+2} \\
\cdots & \cdots \\
c & \underline{b}_{t} \\
0 & \underline{b}_{t+1} \\
\cdots & \cdots \\
0 & \underline{b}_{n}
\end{array}\right)^{\prime}
$$

where the $\underline{b}_{i}$ are appropriate nonnegative $k-1$ vectors satisfying $\underline{b}_{t} \leq \cdots \leq \underline{b}_{s+1}$ and $\underline{b}_{n} \leq \cdots \leq \underline{b}_{t+1}$. Let

$$
a^{\prime}=\left(\begin{array}{cc}
a_{11}-1 & \underline{b}_{1} \\
a_{21}-1 & \underline{b}_{2} \\
\ldots & \ldots \\
a_{s 1}-1 & \underline{b}_{s} \\
c & \underline{0} \\
c & \underline{0} \\
\ldots & \underline{0} \\
c & \underline{b} \\
0 & \underline{b}_{t+1} \\
\ldots & \underline{b}_{n} \\
0 & \underline{b}_{n}
\end{array}\right) \text { and } e=\left(\begin{array}{cc}
1 & \underline{0} \\
1 & \underline{0} \\
\ldots & \ldots \\
1 & \underline{0} \\
0 & \underline{b}_{s+1} \\
0 & \underline{b}_{s+2} \\
\ldots & \ldots \\
0 & \underline{b}_{t} \\
0 & \underline{0} \\
\ldots & \ldots \\
0 & \underline{0}
\end{array}\right) ;
$$

then $a^{\prime}=\max a^{\prime G}$ and $e=\max e^{G}$, and we get $a^{+}-a^{\prime} i^{+} e^{+}=\left(a^{\prime} e\right)^{+}-a^{{ }^{+}} e^{+} \prec a^{+}$. Hence $a^{+}-a^{\prime+} e^{+} \in \mathcal{B}^{1} \cap A_{m d(a)}^{G}+\mathrm{R}(d)$. Obviously $a^{\prime+} e^{+} \in \mathrm{R}(d)$, so we conclude that $a^{+} \in \mathcal{B}^{1} \cap A_{d}^{G}+\mathrm{R}(d)$.

So we can assume that

$$
a=\left(\begin{array}{cc}
c & \underline{b}_{1} \\
c & \underline{b}_{2} \\
\cdots & \ldots \\
c & \underline{b}_{s} \\
0 & \underline{b}_{s+1} \\
0 & \underline{b}_{s+2} \\
\cdots & \ldots \\
0 & \underline{b}_{n}
\end{array}\right)
$$


with $c>1$ and $\underline{b}_{n} \leq \cdots \leq \underline{b}_{s+1}$. Now let

$$
a^{\prime}=\left(\begin{array}{cc}
c-1 & \underline{b}_{1} \\
c-1 & \underline{b}_{2} \\
\cdots & \cdots \\
c-1 & \underline{b}_{s} \\
0 & \underline{0} \\
0 & \underline{0} \\
\ldots & \cdots \\
0 & \underline{0}
\end{array}\right) \quad \text { and } \quad e=\left(\begin{array}{cc}
1 & \underline{0} \\
1 & \underline{0} \\
\ldots & \ldots \\
1 & \underline{0} \\
0 & \underline{b}_{s+1} \\
0 & \underline{b}_{s+2} \\
\ldots & \ldots \\
0 & \underline{b}_{n}
\end{array}\right)
$$

Again $a^{\prime}=\max a^{\prime G}$ and $e=\max e^{G}$, and we conclude as above.

Finally we can assume that

$$
a=\left(\begin{array}{cc}
1 & \underline{b}_{1} \\
1 & \underline{b}_{2} \\
\cdots & \cdots \\
1 & \underline{b}_{s} \\
0 & \underline{b}_{s+1} \\
0 & \underline{b}_{s+2} \\
\cdots & \cdots \\
0 & \underline{b}_{n}
\end{array}\right)
$$

with $\underline{b}_{s} \leq \cdots \leq \underline{b}_{1}$ and $\underline{b}_{n} \leq \cdots \leq \underline{b}_{s+1}$. Now let

$$
a^{\prime}=\left(\begin{array}{cc}
1 & \underline{0} \\
1 & \underline{0} \\
\cdots & \cdots \\
1 & \underline{0} \\
0 & \underline{b}_{s+1} \\
0 & \underline{b}_{s+2} \\
\cdots & \cdots \\
0 & \underline{b}_{n}
\end{array}\right) \quad \text { and } e=\left(\begin{array}{cc}
0 & \underline{b}_{1} \\
0 & \underline{b}_{2} \\
\cdots & \ldots \\
0 & \underline{b}_{s} \\
0 & \underline{0} \\
0 & \underline{0} \\
\cdots & \cdots \\
0 & \underline{0}
\end{array}\right)
$$

Again $a^{\prime}=\max a^{\prime G}$ and $e=\max e^{G}$, and we conclude as above.

If $a_{1}>n$ or $a_{1}=n$ and $m d(a) \neq(n, 0, \ldots, 0)$, then, by definition, $\mathcal{B}^{1} \cap A_{m d(a)}^{G}=0$ and $a^{+}$must be decomposable. If $\operatorname{md}(a)=(n, 0, \ldots, 0)$, then $\mathcal{B}^{1} \cap A_{m d(a)}^{G}=R \underline{1}^{(1)}$. This finishes the proof.

Notice that we also have a right $\Sigma_{k}$-action on $A$ by permuting 'columns' of monomials. On $\mathcal{M}$ this action is described by right multiplication with $k \times n$ permutation matrices. Hence $A$ is a $R \Sigma_{n}-R \Sigma_{k}$-bimodule; in particular $\Sigma_{k}$ also acts on the invariant ring $A^{G}$.

Clearly the total degree of a monomial is not changed by column permutations; hence if $a, b \in \mathcal{M}$ are largest in their $\Sigma_{n}$-orbits and $a$ and $b$ lie in the same $\Sigma_{k}$-orbit, then $a^{+}$is decomposable if and only if $b^{+}$is decomposable. This observation leads to the following result.

Theorem 4.6. For $j=1, \ldots, k$ let $\underline{1}^{(j)} \in \mathcal{M}$ denote the matrix whose entries are all one in the $j$ column and zero elsewhere, and define

$$
\begin{aligned}
\mathcal{M}_{n}:= & \left\{a^{+} \in A^{\Sigma_{n}} \mid \operatorname{md}(a) \text { is dominated by }(n-1, \ldots, n-1)\right\} \\
& \cup\left\{\underline{1}^{(j)} \mid 1 \leq j \leq k\right\} .
\end{aligned}
$$


Then $A^{\Sigma_{n}}=R\left[\mathcal{M}_{n}\right]$. In particular,

$$
\beta\left(V(n, k), \Sigma_{n}\right) \leq \max \{n, k(n-1)\},
$$

i.e. $A^{\Sigma_{n}}$ can be generated by elements of degree $\leq \max \{n, k(n-1)\}$.

Proof. By 4.2 it suffices to show that for each element $a \in \mathcal{M}$ with $a^{+}$not in $\mathcal{M}_{n}$, there is a suitable $f \in R\left[\mathcal{M}_{n}\right]$ with $d(f) \leq d(a)$ or $f=0$ such that $a^{+}-f$ is decomposable. So suppose that $a^{+}$has multidegree $\left(a_{1}, \ldots, a_{n}\right)$ with total degree $d$ and $a_{i} \geq n$. Since $\mathcal{M}_{n}$ and $\mathrm{R}(d)$ are stable under column permutations, we can assume that $i=1$. Now 4.5 tells us that $a^{+} \in\left(\mathcal{B}^{1} \cap A_{m d(a)}^{G}\right)+\mathrm{R}(d)$, and that if $a_{1}>n$ or $a_{1}=n$ and $m d(a) \neq(n, 0, \ldots, 0), a^{+}$must be decomposable. If $m d(a)=(n, 0, \ldots, 0)$, then $a^{+}-r \cdot \underline{1}^{(1)}$ is decomposable for some $r \in R$.

As mentioned in the introduction, we will now show that our new degree bound is 'best possible' in the following sense:

Theorem 4.7. Let $n=p^{s}$ be the power of a prime $p$, and let $R$ be $\mathbb{Z}$ or a ring with $p \cdot 1_{R}=0$. Then

$$
\beta\left(V(n, k), \Sigma_{n}\right)=\max \{n, k(n-1)\} .
$$

Proof. Let $G=\Sigma_{n}$, and let $A_{R}$ denote $A$ if it is defined over $R$. Since $V$ is a permutation module, $A_{R}^{\Sigma_{n}}$ is a free $R$-module, spanned by orbit sums of monomials. Hence $A_{R}^{\Sigma_{n}}=A_{\mathbb{Z}}^{\Sigma_{n}} \otimes_{\mathbb{Z}} R$, and we have $\beta\left(V_{R}(n, k), \Sigma_{n}\right) \leq \beta\left(V_{\mathbb{Z}}(n, k), \Sigma_{n}\right)$. Hence it suffices to show that for each $k \geq 1$ and $R$ with $p \cdot 1_{R}=0$ the element $a_{k}^{+}$with

$$
a_{k}:=\left(\begin{array}{cc}
1 & \underline{0} \\
1 & \underline{0} \\
\cdots & \cdots \\
1 & \underline{0} \\
0 & \underline{b}_{n-1}
\end{array}\right)
$$

and $\underline{b}_{n-1}=(n-1, \ldots, n-1) \in \mathbb{N}_{0}^{k-1}$ is indecomposable in $A(n, k)^{G}$. We use induction on $k$. We have $a_{1}^{+}=e_{n-1}$; notice that $A_{R}(n, 1)^{\Sigma_{n}}=R\left[e_{1}, \ldots, e_{n}\right]$ is a polynomial ring generated by the elementary symmetric polynomials $e_{i}, i=1, \ldots, n$, which are algebraically independent; hence the indecomposability of $a_{1}^{+}$is a consequence of the main theorem on symmetric functions. Assume now that $a_{k-1}^{+}$is indecomposable but $a_{k}^{+}$is not. Then

$$
a_{k}^{+} \in \sum_{\underline{0} \neq \underline{d}_{k}} A(n, k)_{\underline{d}_{k}}^{G} \cdot A(n, k)_{\underline{n-1}_{k}-\underline{d}_{k}}^{G},
$$

where $\underline{n-1}_{k}=(n-1, \ldots, n-1) \in \mathbb{N}^{k}$ and the $\underline{d}_{k}$ are strictly dominated by $\underline{n-1} \underline{1}_{k}$. Now we consider the $G$-equivariant ring epimorphism $s: A_{R}(n, k) \rightarrow A_{R}(n, k-1)$ which is the identity on the $x_{i \ell}$ for $\ell<k$ and maps $x_{i k}$ to 1 for $i=1, \ldots, n$. Notice that for $b \in \mathcal{M}$,

$$
s\left(b^{+}\right)=\frac{\left|G_{s(b)}\right|}{\left|G_{b}\right|}(s(b))_{G}^{+} .
$$

In particular,

$$
s\left(a_{k}^{+}\right)=\frac{\left|G_{a_{k-1}}\right|}{\left|G_{a_{k}}\right|} a_{k-1}^{+}=a_{k-1}^{+} .
$$


It is easy to see from 4.5 that each $h^{+} \in A_{(0, \ldots, 0, i)}^{G}$ with $0<i \leq n-1$ is a linear combination of products of elements of the form $b_{j}^{+}$with

$$
b_{j}=\left(\begin{array}{cc}
\underline{0} & 1 \\
\underline{0} & 1 \\
\cdots & \ldots \\
\underline{0} & 0 \\
\underline{0} & 0
\end{array}\right)
$$

with $0<j<n$ ones and $n-j$ zeros in the last column. We get $G_{s\left(b_{j}\right)}=G$, and hence

$$
\frac{\left|G_{s\left(b_{j}\right)}\right|}{\left|G_{b_{j}}\right|}=\left|b_{j}^{G}\right|=\left(\begin{array}{c}
n \\
j
\end{array}\right) \equiv 0 \bmod (p)
$$

by Lucas' congruences; hence $s\left(h^{+}\right)=0$ for each $h^{+} \in A_{(0, \ldots, 0, i)}^{G}$ with $0<i<n$.

Clearly we have $s\left(A(n, k)_{\underline{d}_{k}}^{G}\right) \subseteq A(n, k-1)_{\underline{d}_{k-1}}^{G}$; hence

$$
a_{k-1}^{+}=s\left(a_{k}^{+}\right) \in \sum_{\underline{0} \neq \underline{d}_{k-1}} A(n, k-1)_{\underline{d}_{k-1}}^{G} \cdot A(n, k-1)_{\underline{n-1}_{k-1}-\underline{d}_{k-1}}^{G} .
$$

Since $s\left(A(n, k)_{(0, \ldots, 0, i)}^{G}\right)=0$ for $0<i \leq n-1$, all 'surviving' summands must have $\underline{d}_{k-1}=\left(d_{1}, \ldots, d_{k-1}\right)$ different from $(0, \ldots, 0)$ and strictly dominated by $(n-1, \ldots, n-1)$. But this implies that $a_{k-1}^{+}$is decomposable, which is a contradiction.

\section{ACKNowledGement}

I thank the referee for carefully reading the manuscript and for valuable suggestions, which improved the exposition of the paper.

\section{REFERENCES}

[1] H.E.A. Campbell, I. Hughes, R.D. Pollack, Vector invariants of symmetric groups, Canad. Math. Bull. 33, 391-397, (1990). MR 92g:13004

[2] S. Hu, M. Kang, Efficient generation of the ring of invariants, J. Algebra, 180, 341-364, (1996). MR 97b:13006

[3] E. Noether, Der Endlichkeitssatz der Invarianten endlicher Gruppen, Math. Ann. 77, 89-92, (1916).

[4] E. Noether, Der Endlichkeitssatz der Invarianten endlicher linearer Gruppen der Charakteristik p, Nachr. Ges. Wiss. Göttingen (1926), 28-35; reprinted in 'Collected Papers', pp. 485-492, Springer Verlag, Berlin (1983).

[5] D. Richman, Explicit generators of the invariants of finite groups, Adv. Math. 124 (1996), 49-76. CMP 97:05

[6] L. Smith, E. Noether's bound in the invariant theory of finite groups, Arch. Math. 66, 89-92, (1995). MR 96k:13004

[7] L. Smith, Polynomial Invariants of Finite Groups, A.K. Peters Ltd., (1995). MR 96f:13008

[8] H. Weyl, The Classical Groups, 2nd ed., Princeton Univ. Press, Princeton (1953). MR 1:42c (1st ed.)

Institute for Experimental Mathematics, University of Essen, Ellernstr. 29, 45326 Essen, Germany

E-mail address: peter@exp-math.uni-essen.de 